\title{
Table of Contents - Volume One
}

INTRODUCTION

xiii

Acknowledgments

xiv

Institutional Abbreviations

Methods and Terminology. By EUGENIA B. BöHLKE

Phylogenetic Relationships of Anguilliform Fishes. By C. RICHARD ROBINS

$x \mathbf{v}$

Order Anguilliformes

Family Anguillidae.

By DAVID G. SMITH

Genus Anguilla

Family Heterenchelyidae. By DAvid G. SMITH 48

Genus Pythonichthys

Family Moringuidae. By DAvID G. SMITH

Genus Neoconger

Genus Moringua

Family Chlopsidae. By David G. SMITH $\quad 72$

Genus Linkenchelys

Genus Robinsia

Genus Catesbya

Genus Chilorhinus

Genus Kaupichthys

Genus Chlopsis

Family Myrocongridae. By David G. SMITH $\quad 98$

Genus Myroconger

Family Muraenidae. By EUGENIA B. BöHLKE, JOHN E. MCCOSKER, and JAMES E. BÖHLKE

Subfamily Uropterygiinae

Genus Anarchias

Genus Channomuraena

Genus Uropterygius

Subfamily Muraeninae

Genus Echidna

Genus Enchelycore

Genus Gymnothorax

Genus Monopenchelys

Genus Muraena

Family Synaphobranchidae. By CATHERINE H. Robins and C. RICHARD RoBINS

Subfamily Simenchelyinae

Genus Simenchelys 
Subfamily Synaphobranchinae $\quad 218$

Genus Synaphobranchus $\quad 219$

Genus Haptenchelys $\quad 232$

Subfamily Ilyophinae $\quad 234$

Genus Ilyophis $\quad 235$

Genus Meadia $\quad 239$

Genus Atractodenchelys $\quad 240$

Genus Dysommina $\quad 242$

Genus Dysomma

Family Ophichthidae. By JOHN E. MCCOSKER, EuGENIA B. BÖHLKE,
and JAMES E. BöHLKE

Subfamily Myrophinae $\quad 270$

Genus Ahlia $\quad 272$

Genus Asarcenchelys $\quad 276$

Genus Mixomyrophis $\quad 277$

Genus Myrophis $\quad 279$

Genus Pseudomyrophis $\quad 289$

Subfamily Ophichthinae $\quad 296$

Genus Aprognathodon $\quad 300$

Genus Callechelys $\quad 303$

Genus Letharchus $\quad 313$

Genus Apterichtus $\quad 317$

Genus Ichthyapus $\quad 322$

Genus Stictorhinus $\quad 325$

Genus Bascanichthys $\quad 329$

Genus Caralophia $\quad 338$

Genus Ethadophis 341

Genus Gordiichthys

Genus Phaenomonas 351

Genus Aplatophis $\quad 354$

Genus Echiophis

Genus Herpetoichthys

Genus Hyphalophis $\quad 367$

Genus Kertomichthys $\quad 368$

Genus Lethogoleos $\quad 370$

Genus Myrichthys $\quad 372$

Genus Ophichthus $\quad 379$

$\begin{array}{ll}\text { Genus Quassiremus } & 409\end{array}$

Family Colocongridae. By DAVID G. SMITH

Genus Coloconger $\quad 417$

Family Derichthyidae. By CATHERINE H. RobINS $\quad 420$

Genus Derichthys $\quad 424$

Genus Nessorhamphus $\quad 427$

Family Muraenesocidae. By DAvID G. SMITH 432

Genus Cynoponticus $\quad 436$

Family Nemichthyidae. By DAvid G. SMITH

Genus Avocettina $\quad 447$ 
Genus Labichthys

Genus Nemichthys

Family Congridae. By David G. SMITH $\quad 460$

Subfamily Heterocongrinae $\quad 482$

Genus Heteroconger

484

Subfamily Bathymyrinae $\quad 490$

Genus Ariosoma $\quad 491$

Genus Parabathymyrus $\quad 503$

Genus Paraconger $\quad 506$

Subfamily Congrinae $\quad 511$

Genus Conger $\quad 513$

Genus Gnathophis $\quad 521$

Genus Rhynchoconger $\quad 525$

Genus Rhechias $\quad 532$

Genus Bathyuroconger $\quad 541$

Genus Uroconger $\quad \mathbf{5 4 5}$

Genus Japonoconger $\quad 549$

Genus Pseudophichthys

Genus Acromycter $\quad 554$

Genus Xenomystax $\quad 558$

Family Nettastomatidae. By DAvID G. SMITH 568

Genus Hoplunnis $\quad 576$

Genus Saurenchelys $\quad \mathbf{5 9 0}$

Genus Facciolella $\quad 596$

Genus Nettenchelys $\quad 599$

Genus Nettastoma $\quad 604$

$\begin{array}{ll}\text { Genus Venefica } & 609\end{array}$

Family Serrivomeridae. By KeNNETH A. TIGHE 613

$\begin{array}{ll}\text { Genus Serrivomer } & 617\end{array}$

Genus Stemonidium $\quad 625$

Order Saccopharyngiformes. By DAvID G. SMITH 629

Family Cyematidae. By DAvid G. SMITH $\quad 630$

Genus Cyema $\quad 633$

Families Saccopharyngidae, Eurypharyngidae, and Monognathidae.

By E. Bertelsen, Jorgen G. Nielsen, and David G. Smith 636

Family Saccopharyngidae $\quad 642$

Genus Saccopharynx $\quad 643$

Family Eurypharyngidae $\quad 648$

Genus Eurypharynx $\quad 649$

Family Monognathidae $\quad 650$

Genus Monognathus $\quad 652$ 
This page intentionally left blank 